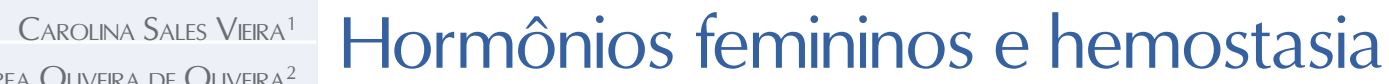

\section{Female hormones and hemostasis}

\section{Artigos de revisão}

Palavras-chave

Tromboembolismo

Hemostasia

Contraceptivos orais

Terapia de reposição hormonal

Keywords

Thromboembolism

Hemostasis

Contraceptives, ora

Hormone replacement therapy

\section{Resumo}

Os hormônios femininos exógenos utilizados para contracepção ou para terapia hormonal (TH) no climatério estão associados a aumento de risco para tromboembolismo venoso (TEV), principalmente por provocarem alterações prócoagulantes na hemostasia. Este risco não é cumulativo e parece ser maior no primeiro ano de uso. A dose de estrogênio, o tipo de estrogênio e progestagênio utilizados, a via de administração hormonal e os fatores de risco hereditários para trombose venosa de cada paciente interferem no risco final para trombose venosa. $\bigcirc$ conhecimento dos efeitos na hemostasia inerentes a cada composto hormonal é essencial para uma prescrição adequada.

\section{Abstract}

Exogenous female hormones used for contraception or postmenopausal hormonal replacement therapy are associated with an increase of venous thromboembolism (VTE) risk, mainly because they cause a hypercoagulable state. The risk is highest during the first year of use and it is not cumulative. The dose of estrogen, the type of estrogen and progestogen, the route of administration of female sex steroid hormones, and the hereditary risk factors for VTE of each patient can interfere on the final risk for VTE. The knowledge of their effect on hemostasis is essential for a correct prescription.

Correspondência:

Carolina Sales Vieiro Departamento de Ginecologia e Obstetrícia da Faculdade de Medicina de Ribeirão Preto da Universidade de São Paulo - USP Ribeirão Preto (SP), Brasil

Avenida Bandeirantes, 3.900 - Campus Universitário CEP 14049-900 - Ribeirão Preto/SP

Fone: (16) 3602-2804

Fax: (16) 3633-0946

E-mail: carol.sales@uol.com.br

Recebido

28/08/2007

Aceito com modificacōos

08/10/2007
${ }^{1}$ Médica Assistente do Setor de Reprodução Humana do Departamento de Ginecologia e Obstetrícia da Faculdade de Medicina de Ribeirão Preto da Universidade de São Paulo - USP - Ribeirão Preto (SP), Brasil.

${ }^{2}$ Médica Assistente do Setor de Hemostasia do Departamento de Clínica Médica da Faculdade de Medicina de Ribeirão Preto da Universidade de São Paulo - USP - Ribeirão Preto (SP), Brasil.

${ }_{3}^{3}$ Professor Titular do Setor de Reprodução Humana do Departamento de Ginecologia e Obstetrícia da Faculdade de Medicina de Ribeirão Preto da Universidade de São Paulo - USP - Ribeirão Preto (SP), Brasil. 


\section{Introdução}

Vários estudos epidemiológicos têm demonstrado uma associação clara entre o uso de contraceptivos orais combinados (COCs) e o aumento de risco para trombose venosa e arterial $^{1-3}$. Apesar de as tromboses venosas e arteriais possuírem alguns fatores de riscos em comum para sua ocorrência, sabe-se que a estase sangüínea e a hipercoagulabilidade representam os principais fatores etiopatogênicos para o desencadeamento do tromboembolismo venoso (TEV), enquanto que a lesão do endotélio (principalmente pela formação de aterosclerose) representa a principal determinante da trombose arterial (infarto do miocárdio, acidente vascular cerebral e doença arterial periférica), conforme Tabela 1. Vale ressaltar que a trombose arterial é menos freqüente no menacme que o TEV (um caso de trombose arterial para cada 5-10 casos de TEV) ${ }^{4}$.

Assim, o objetivo desta revisão é a discussão dos principais efeitos dos esteróides sexuais na hemostasia, bem como estratégias para redução do risco de trombose venosa, que representa o efeito adverso mais temido do uso de COCs e que está diretamente ligada a quadros de alterações pró-coagulantes da hemostasia.

\section{Sistema hemostático}

$\mathrm{O}$ adequado funcionamento do sistema circulatório depende de uma série de mecanismos que regulam a manutenção do sangue no estado fluido dentro do compartimento vascular, permitindo a perfusão adequada a todos

Tabela 1 - Fatores predisponentes para desenvolvimento de trombose.

\begin{tabular}{|c|c|c|c|}
\hline Tipo de trombose & Lesc̃o no endotélio & Hipercoagulabilidade & Estase \\
\hline Venosa & $\begin{array}{c}\text { Idade } \\
\text { Passado de TEV }\end{array}$ & $\begin{array}{c}\downarrow \text { antitrombina } \\
\downarrow \text { proteínas C ou S } \\
\text { Fator V Leiden } \\
\text { Protrombina G20210A } \\
\text { SAAF } \\
\text { Disfibrinogenemia } \\
\uparrow \text { protrombina } \\
\uparrow \text { níveis Fator VIII, } \\
\text { IX e XI } \\
\text { Gravidez } \\
\text { Neoplasia maligna } \\
\text { COC e TH }\end{array}$ & $\begin{array}{c}\text { Idade } \\
\text { Passado de TEV } \\
\text { Cirurgia } \\
\text { Gravidez } \\
\text { Puerpério } \\
\text { Imobilização } \\
\text { Viagem } \\
\text { prolongada }\end{array}$ \\
\hline Arterial & $\begin{array}{l}\text { Idade } \\
\text { Tabagismo } \\
\text { HAS } \\
\text { Hipercolesterolemia } \\
\text { Diabetes mellitus } \\
\text { Sedentarismo }\end{array}$ & $\begin{array}{c}\text { COC } \\
\text { SAAF } \\
\text { Hiperhomocisteinemia } \\
\text { Fator V Leiden (?) } \\
\text { Protrombina G20210A } \\
\text { (?) } \\
\uparrow \text { níveis Fator VIII (?) } \\
\uparrow \text { níveis Fator IX (?) }\end{array}$ & - \\
\hline
\end{tabular}

(Traduzida e Adaptada de Rosendaal et al. 0 ).

$\mathrm{SAAF}=$ síndrome do anticorpo anti-fosfolípides; TEV=tromboembolismo venoso; $\mathrm{HAS}=$ hipertensão arterial sistêmica; $C O C=$ contraceptivo oral combinado; $\mathrm{TH}=$ terapia hormonal. os territórios do organismo. Os componentes do sistema hemostático incluem as plaquetas, os vasos sanguíneos, o fator de von Willebrand, os fatores da coagulação, os anticoagulantes naturais e o sistema fibrinolítico. $\mathrm{Na}$ vigência de qualquer lesão vascular, esses componentes são ativados, visando à manutenção da integridade do endotélio e evitando a perda excessiva de sangue.

A ativação dos fatores da coagulação, de acordo com um modelo didaticamente apresentado como "cascata", culmina com a formação de um tampão hemostático, constituído de plaquetas e fibrina, no local da lesão vascular. A formação do tampão de plaqueta e fibrina deve-se manter restrita ao sítio de lesão endotelial, de forma a prevenir a coagulação disseminada e a doença tromboembólica. Os anticoagulantes naturais, cujos principais representantes são a antitrombina e as proteínas $\mathrm{C}$ e $\mathrm{S}$, atuam principalmente por meio da degradação de fatores da coagulação. Já o sistema fibrinolítico atua sobre a fibrina formada no local da lesão vascular, degradando-a e estabilizando o coágulo. Desta forma, em condições normais, os mecanismos anticoagulantes prevalecem sobre os pró-coagulantes (Figura 1) . $^{5}$.

\section{Estrogênios e hemostasia}

O uso de COC está associado a um aumento de duas a seis vezes no risco para desenvolvimento de $\mathrm{TEV}^{1,6}$. Este risco aumentado para TEV foi primariamente associado ao componente estrogênico dos COCs de forma dose-dependente, ocasionando queda gradual

A

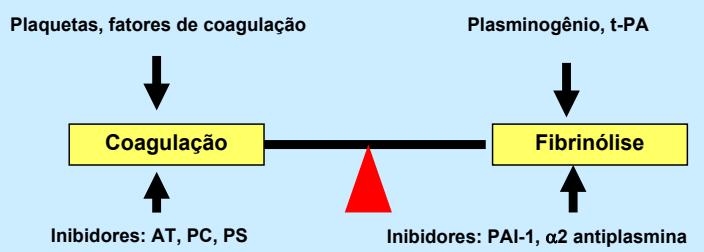

B

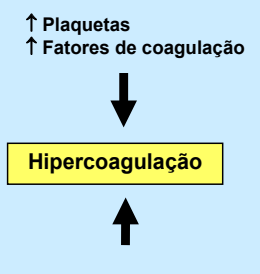

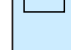

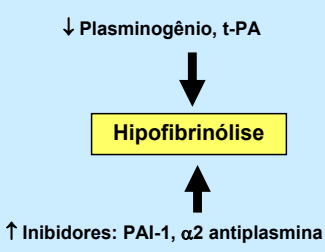

$\downarrow$ Inibidores: AT, PC, PS

AT=antitrombina; $P C=$ proteína $C ; P S=$ proteína $S ; t-P A=$ ativador do plasminogênio

tissular; PAl=inibidor do ativador do plasminogênio (Traduzida e Adaptada de Conard, 199923.

Figura 1 - (A) Hemostasia normal; (B) Hemostasia alterada: mecanismo de trombose. 
da quantidade de etinilestradiol (EE) (de $100 \mu \mathrm{g}$ para 20-15 $\mu \mathrm{g}$ ) nos COCs.

Foi demonstrado que a alta dosagem de EE ( $\geq 50 \mathrm{mcg}$ ) está associada a um aumento de duas vezes no risco de TEV quando comparada à baixa dosagem deste hormônio ( $<50 \mathrm{mcg}$ ), porém as formulações com ultra-baixa dose de EE (15 e 20 mcg) não mostraram redução significativa de risco para $\mathrm{TEV}^{6,7}$. No entanto, ainda não há estudos publicados com amostra suficiente para determinar se existe diferença no risco de TEV entre COCs de baixa dosagem e aqueles com ultra-baixa dose de EE, mas está sendo conduzido um estudo multicêntrico, coordenado pela Universidade de Leiden, Holanda, chamado Multiple Environmental and Genetics Assessment of Risk Factors for Venous Thrombosis (MEGA) ${ }^{6}$, que tem como um dos objetivos responder a esta questão.

O EE induz alterações significativas no sistema de coagulação, culminando com aumento da geração de trombina. Conforme mostrado na Tabela 2, ocorre também aumento dos fatores de coagulação (fibrinogênio, VII, VIII, IX, X, XII e XIII) e redução dos inibidores naturais da coagulação (proteína $\mathrm{S}$ e antitrombina), produzindo um efeito pró-coagulante leve ${ }^{8,9}$. Estes efeitos são mais claramente observados em testes que avaliam globalmente a hemostasia, que mostram resistência adquirida à proteína $\mathrm{C}$ (marcador de resistência à ação do anticoagulante natural - proteína C) e aumento de geração de trombina?.

Para terapia hormonal (TH) no climatério, acreditavase, inicialmente, que, pelo uso de estrogênios similares ao natural, não haveria aumento de risco para trombose

Tabela 2 - Efeitos dos contraceptivos orais combinados na hemostasia.

\begin{tabular}{ll}
\hline Pró-coagulante & Pró-fibrinolítico \\
\hline$\uparrow$ fibrinogênio & $\uparrow$ plasminogênio \\
$\uparrow$ fatores VIII, VIII, IX, X, XII, XIII & $\downarrow$ PAl \\
$\downarrow$ proteína $S$ e antitrombina & \\
Resistência adquirida à proténa C ativada & \\
\hline
\end{tabular}

(Traduzida e Adaptada de Mammen, $2000^{8}$ e Rosendaal et al., 2005\%). PAl=inibidor do ativador do plasminogênio.

Tabela 3 - Risco absoluto para ocorrência de tromboembolismo venoso.

\begin{tabular}{lll}
\hline Idade (anos) & Risco absoluto anual & $\begin{array}{l}\text { Risco com COC, } \\
\text { Gravidez e TRH }\end{array}$ \\
\hline$<30$ annos & $5 / 100.000$ & $\begin{array}{l}\text { COC } 15-30 / 100.000 \\
\text { Gravidez 50-75/100.000 }\end{array}$ \\
50 annos & $1 / 10.000$ & TRH oral 3/10.000 \\
$60-70$ annos & $1 / 1.000$ & TRH oral 3/1.000 \\
$>80$ annos & $1 / 100$ & Não aplicável \\
\hline
\end{tabular}

(Traduzida e Adaptado de Lowe, 2004 ${ }^{14}$ ).

$\mathrm{COC}=$ contraceptivo oral combinado, $\mathrm{TRH}=$ terapia de reposição hormonal. venosa e arterial. No entanto, surgiram vários estudos que confirmaram um aumento de três vezes no risco para TEV em usuárias de TH comparado a não-usuárias ${ }^{10-13}$. É importante salientar que a população usuária de TH, pela própria idade, já apresenta risco basal para TEV dez vezes superior às usuárias de COC. Mesmo com este risco relativo basal elevado, devemos lembrar que o risco absoluto é baixo (três casos de TEV em 10.000 usuárias de TH), conforme Tabela $3^{9,14}$.

Em 2004, foi demonstrado que o tipo de estrogênio utilizado em TH tem influencia no risco para TEV. O uso de estrogênios eqüinos conjugados (EEC) está associado a aumento de risco para TEV comparado aos estrogênios esterificados - um estrogênio não disponível no Brasil [EEC: $\mathrm{OR}=1,7 ; \mathrm{IC95 \% =1,2-2,2)} \mathrm{versus} \mathrm{estrogênios}$ esterificados $=\mathrm{OR}=0,9$; IC95\% $=0,7-1,2)]^{15}$. Esta diferença de risco foi atribuída ao fato de o EEC produzir $52 \%$ mais resistência à proteína $\mathrm{C}$ ativada do que os estrogênios esterificados, o que culmina em maior produção de trombina pelo EEC do que pelo último ${ }^{16}$. Em portadoras de trombofilias hereditárias, que apresentam risco basal elevado para TEV, o uso de EEC também produziu mais eventos trombóticos do que o uso de estrogênios esterificados, sendo que esta última formulação não aumentou significativamente o risco basal das voluntárias trombofílicas ${ }^{17}$. Estes dados não significam ainda que se deva usar de rotina os estrogênios esterificados em pacientes trombofílicas, mas que há opções menos trombogênicas e, quando indicada a $\mathrm{TH}$, estas devem ser preferidas ao EEC, mesmo em pacientes sem risco aumentado para TEV. Estudos randomizados são necessários para confirmar se realmente os estrogênios esterificados são de fato associados à ausência de risco para trombose, uma vez que todos estes resultados advêm de estudos observacionais tipo caso-controle. Também são necessárias comparações do EEC com o 17-beta-estradiol e o valerato de estradiol para identificar outras opções orais com menor risco de TEV.

\section{Progestagênios e hemostasia}

Inicialmente, achava-se que a trombose era resultado apenas da dose de estrogênio utilizado. Porém, em 1995, demonstrou-se que COCs contendo progestagênios de terceira geração (gestodeno, desogestrel) associavam-se a um risco duas vezes maior de trombose do que os COCs que continham progestagênios de segunda geração (levonorgestrel) ${ }^{1,2}$. Esse resultado estimulou pesquisas e debates que tentavam explicar se o risco elevado para TEV era devido à ação dos progestagênios envolvidos, às características das usuárias ou aos vieses apontados nesses estudos. Posteriormente, uma meta-análise avaliou os COCs contendo progestagênios 
de terceira geração e o risco para TEV, confirmando que esses progestagênios associavam-se a um risco para TEV 1,7 vezes maior que o risco dos progestagênios de segunda geração $^{18}$. Dessa forma, o tipo de progestagênio associado ao estrogênio, e não só a dose deste último, tornou-se motivo de estudos sobre o papel dos progestagênios na hemostasia e na determinação da trombose.

\section{Associações estro-progestagênicas}

Os progestagênios formam um grupo de esteróides que, apesar de possuírem a característica comum de se ligarem aos receptores de progesterona, têm efeitos sistêmicos diferentes e que são mediados não só pela afinidade aos próprios receptores de progesterona, mas principalmente pela capacidade de ligação com os receptores de outros esteróides, como os estrogênios, androgênios, glicocorticóides e mineralocorticóides ${ }^{19}$. Essa capacidade de ligar-se a outros receptores de esteróides, bem como o perfil de afinidade por cada um desses receptores, pode resultar em riscos diferentes para a trombose, a depender do progestagênio associado ao estrogênio. Um exemplo dessa afirmação é um estudo experimental que comparou o efeito pró-coagulante em vasos sangüíneos tratados com diversos progestagênios isolados, mostrando maior expressão de receptores para trombina, quando foram usados progestagênios com maior atividade glicocorticóide ${ }^{20}$.

Com a polêmica gerada pelos relatos da existência de maior risco para TEV em usuárias de COCs que continham progestagênios de terceira geração em relação àqueles com segunda geração, houve um estímulo para a descoberta de um mecanismo biológico plausível que explicasse o motivo dessa associação, já que muitas mulheres se beneficiariam de progestagênios com menor efeito androgênico e mineralocorticóide.

COCs com progestagênios de terceira geração estão associados ao desenvolvimento de resistência adquirida à proteína $C$ ativada mais pronunciada ${ }^{21,22}$ e a uma tendência de produzir níveis mais altos de fatores de coagulação e níveis mais baixos de antitrombina e de proteína $S$, quando comparados a COCs contendo progestagênio de segunda geração $0^{9,22-24}$. Esses achados poderiam explicar as observações epidemiológicas de risco aumentado para TEV em usuárias de COCs que contêm progestagênios de terceira geração, já que a resistência à ação da proteína $\mathrm{C}$ (adquirida ou herdada) é um marcador importante para risco aumentado de TEV ${ }^{25}$.

Outro achado é que a hiperfibrinólise é menos acentuada em usuárias de COCs que contêm progestagênios de terceira geração do que aqueles com progestagênios de segunda geração ${ }^{23}$, provavelmente devido à modulação do progestagênio sobre o efeito pró-fibrinolítico do estrogênio nos COCs. Dessa forma, os progestagênios de terceira geração inibiriam com maior intensidade a fibrinólise (lise do coágulo de fibrina) induzida pelo estrogênio do que os progestagênios de segunda geração, podendo também ser outra evidência a corroborar com os dados epidemiológicos encontrados sobre o tipo de progestagênio nos COCs e o risco para TEV.

Outros progestagênios também foram estudados quanto ao risco de trombose quando associados ao EE. Foi demonstrado aumento de quatro vezes no risco para TEV com uso de acetato de ciproterona associado ao EE comparado ao das usuárias de COCs com levonorgestrel ${ }^{26}$, enquanto outro estudo não achou diferença no risco de trombose com estas mesmas drogas ${ }^{27}$. Os resultados preliminares do estudo MEGA, comentado anteriormente, mostrou aumento de 18 vezes no risco para TEV em usuárias de COC contendo acetato de ciproterona comparado a não-usuárias de $\mathrm{COCs}^{6}$. Este resultado pode dever-se ao aumento da resistência à proteína $C$ ativada promovida pelos COCs contendo ciproterona comparados àqueles com levonorgestrel ${ }^{28,29}$.

Avaliando os dados disponíveis na literatura, nota-se que quanto mais efeito androgênico (ou menor poder estrogênico) tem o progestagênio associado ao EE, menor o risco de trombose a que esse COC está associado. Assim, os COCs com levonorgestrel têm menor risco para TEV do que aqueles com desogestrel ou gestodeno, uma vez que esses últimos são menos androgênicos que o primeiro. Da mesma forma, a associação com acetato de ciproterona seria a mais trombogênica, uma vez que é a que contém o progestagênio com maior poder antiandrogênico ${ }^{30,31}$ (Tabela 4).

Recentemente, foi lançado no mercado um COC contendo progestagênio com efeitos antiandrogênio e antimineralocorticóide, a drospirenona ${ }^{19}$. Quando comparado ao levonorgestrel, este se mostrou menos sensível à proteína $C$ ativada (resistência à ação da proteína $C$ ativada), o que poderia predizer um aumento do risco de TEV associado ao seu uso ${ }^{29}$. Outros autores demonstraram efeitos na hemostasia similares entre COCs contendo $20 \mathrm{mcg}$ de $\mathrm{EE}+3 \mathrm{mg}$ de drospirenona e $20 \mathrm{mcg}$ de EE $+150 \mathrm{mcg}$ de desogestrel ${ }^{32}$. Apesar destas informações, o risco de TEV foi similar ao das usuárias de COC contendo levonorgestrel ${ }^{33}$. Entretanto, mais estudos e maior tempo de uso são necessários para uma conclusão definitiva sobre o risco de TEV entre usuárias de COC contendo drospirenona.

Apesar dos dados apresentados, não se quer dizer que o ginecologista deva apenas usar o levonorgestrel como progestagênio associado ao EE, mas que deve conhecer os riscos de TEV, bem como os benefícios adicionais de cada progestagênio, para uma prescrição adequada aos 
anseios e características clínicas da paciente. Além disso, o ciclo gravídico-puerperal apresenta maior risco de TEV do que qualquer formulação contraceptiva apresentada. Entretanto, quando se estima que há no mundo cerca de 100 milhões de mulheres em uso de contracepção hormonal ${ }^{34}$, o conhecimento de opções menos trombogênicas torna-se de grande importância.

Se, para contracepção parece haver menor risco para TEV quanto mais androgênico for o progestagênio associado ao estrogênio, para $\mathrm{TH}$ no climatério ainda dispomos de poucas evidências a respeito do progestagênio com menor risco para TEV. Em estudo recente, tipo caso-controle, a progesterona micronizada e os derivados pregnanos (diidrogesterona, clormadinona) tiveram menor risco de TEV do que os norpregnanos (acetato de nomegestrol e promesgestrona $)^{35}$, porém, apesar de ser um estudo com desenho adequado, a amostra ainda é pequena para uma conclusão definitiva.

Desta maneira, observa-se que tanto em contracepção quanto em TH no climatério, o progestagênio, provavelmente por sua capacidade de interação com receptores de outros esteróides, é capaz de modular o efeito do estrogênio na hemostasia, alterando o risco para TEV a depender não só da dose de estrogênio utilizado, mas também do progestagênio escolhido para associação. Obviamente, por ser um evento raro, não precisamos deixar de prescrever um ou outro COC, mas também não devemos prescrever COCs com alto potencial trombogênico a pacientes de risco para TEV ou mesmo para aquelas de baixo risco sem indicação clínica para um progestagênio de maior impacto sobre a hemostasia.

\section{Progestagênios isolados}

Os progestagênios administrados isoladamente afetam de forma mínima o sistema de coagulação. Um aumento modesto e não significativo no risco para TEV tem sido relatado em usuárias de pílulas contraceptivas somente de progestagênio ${ }^{34,36}$. Os anticoncepcionais com progestagênios isolados não são associados a alterações marcantes nos parâmetros de coagulação e fibrinólise, podendo, por isso, ser indicados para pacientes com risco para $\mathrm{TEV}^{14,37}$.

Um estudo randomizado, duplo-cego, comparando contraceptivos orais compostos apenas de progestagênios (desogestrel versus levonorgestrel) avaliou os efeitos desses nos fatores de coagulação, anticoagulantes naturais e sistema fibrinolítico em 78 mulheres saudáveis, mostrando que ambos apresentaram efeito favorável sobre a hemostasia, reduzindo de forma significativa a atividade do fator VII. Além disso, o desogestrel também provocou um aumento significativo na concentração da proteína $\mathrm{S}^{38}$. Outro estudo recente também mostrou resultados favoráveis com as pílulas de progestagênios (desogestrel versus levonorgestrel), com redução da resistência à proteína $\mathrm{C}$ ativada e aumento da proteína $\mathrm{S}^{22}$. Dessa forma, os efeitos negativos dependentes do tipo de progestagênio provocados pelos COCs sobre os parâmetros de coagulação e anticoagulação não foram observados com o uso de pílulas somente de progestagênio, seja de levonorgestrel ou de desogestrel.

Nos últimos 20 anos, têm sido desenvolvidos implantes subdérmicos que mantêm liberação de baixas doses de progestagênios, com vantagens para pacientes que não toleram a via oral ou se esquecem da ingestão diária de pílulas. Os implantes liberadores de levonorgestrel $\left(\right.$ Norplant $^{\circledR}$, Jadelle $^{\circledR}$ ) foram os primeiros desenvolvidos e, em relação à hemostasia, os dados científicos publicados são escassos, porém são consistentes com os dados de outros contraceptivos com progestagênios que apresentaram mínimo ou nenhum efeito sobre a hemostasia ${ }^{39}$. Soma-se a estes resultados o fato de não terem sido encontrados riscos aumentados com esse contraceptivo para TEV, para infarto agudo do miocárdio e para acidente vascular cerebral quando comparados aos das usuárias de métodos não hormonais e ao número desses eventos esperados na população geral ${ }^{40,41}$.

Outro implante disponível que contém etonogestrel, o metabólito ativo do desogestrel, também não mostrou efeito pró-coagulante. As variáveis hemostáticas analisadas

Tabela 4 - Risco para tromboembolismo venoso associado a cada tipo de contraceptivo.

\begin{tabular}{|c|c|c|c|}
\hline Grupo & $\begin{array}{l}\text { Risco absoluto para TEV } \\
\text { (casos/expostas) }\end{array}$ & $\begin{array}{c}\text { Risco relativo comparado } \\
\text { a não-usuárias }\end{array}$ & $\begin{array}{l}\text { Risco relativo comparado } \\
\text { a usuárias de COCs com LNG }\end{array}$ \\
\hline Risco basal (não-usuárias) & 1 a $5 / 100.000$ & 1 & Não se aplica \\
\hline Progestagênios isolados & Igual ao das não-usuárias & 1 & Não se aplica \\
\hline $\mathrm{COCs}$ com levonorgestrel & 3 a 15/100.000 & 3 & 1 \\
\hline COCs com desogestrel ou gestodeno & 6 a $30 / 100.000$ & 6 & 2 \\
\hline COCs com acetato de ciproterona & 12 a $60 / 100.000$ & $12-18$ & 4 \\
\hline Gestação & 50 a $75 / 100.000$ & 15 & Não se aplica \\
\hline
\end{tabular}

LNG=levonorgestrel, TEV=tromboembolismo venoso, $C O C=$ contraceptivo oral combinado. 
ou não se modificaram ou sofreram alterações discretas, sempre dentro dos valores de normalidade para os ensaios realizados ${ }^{42-44}$, com exceção de um marcador de geração de trombina (o complexo trombina-antitrombina), que reduziu abaixo dos níveis de normalidade, o que é um efeito favorável para hemostasia ${ }^{45}$.

Em TH no climatério, o único progestagênio utilizado é a tibolona, que tem capacidade de ligar-se aos receptores de estrogênio, androgênio e progesterona. Seus efeitos na hemostasia parecem ser menores que a TH em dose convencional ${ }^{46-49}$, porém não há concordância se a tibolona é mais deletéria ou benéfica para hemostasia que a TH em baixas doses ${ }^{46-48}$. No entanto, não há evidência suficiente para afirmar que o risco de TEV em usuárias de tibolona é menor do que em usuárias de TH estro-progestagênica. Assim, em pacientes com trombose prévia ou trombofilia não há indicação de uso da tibolona.

Em resumo, o uso de contraceptivos hormonais apenas com progestagênio não produz um padrão prótrombótico na hemostasia e não aumenta o risco para TEV, sendo, desta forma, indicados para contracepção em pacientes de alto risco para TEV ou com história prévia de trombose (categoria 2 pela Organização Mundial de Saúde (OMS) - os benefícios superam os riscos) $)^{37}$. Já para $T H$, não podemos extrapolar os dados de contracepção e, até o presente momento, não temos evidências para indicar o uso de tibolona para pacientes de alto risco para TEV.

\section{Via de administração de hormônios esteróides e risco para trombose}

Com relação ao risco de TEV, a via de administração hormonal para TH no climatério é importante. Isto porque as evidências atuais sugerem que a via transdérmica parece não aumentar o risco de $\mathrm{TEV}^{35,50}$ comparado à via oral, mesmo com a adição do progestagênio ${ }^{50}$.

Já em contracepção, não podemos afirmar que a via transdérmica tem menor risco de TEV do que a via oral, principalmente porque é utilizado o EE, que tem uma potência superior aos estrogênios utilizados em TH. Em novembro de 2005, o US Food and Drug Administration (FDA) divulgou um comunicado de que o adesivo contendo $0,75 \mathrm{mg}+6 \mathrm{mg}$ de norelgestromin (20 mcg de EE + $150 \mathrm{mg}$ de norelgestrolmin/dia) liberava $60 \%$ a mais do que a quantidade total de EE disponível em um COC de $35 \mathrm{mcg}$ de $\mathrm{EE}^{51}$, o que poderia alterar a freqüência de eventos adversos sérios, como o TEV. Posteriormente, foi realizado um estudo que demonstrou que o adesivo com liberação diária de $20 \mathrm{mcg}$ de EE tem incidência de TEV similar a de um COC com
35 mcg de EE, o que significa que não devemos usar este método para pacientes de alto risco para TEV, ao contrário de $\mathrm{TH}$ transdérmica ${ }^{52}$.

Outros métodos de contracepção hormonal combinada não oral, como o anel vaginal e os injetáveis mensais, foram testados quanto aos seus efeitos na hemostasia. O anel $(15 \mathrm{mcg}$ de $\mathrm{EE}+120 \mathrm{mcg}$ de etonogestrel/dia) foi comparado ao COC contendo $30 \mathrm{mcg}$ de $\mathrm{EE}+150 \mathrm{mcg}$ de levonorgestrel, mostrando alterações similares na hemostasia ${ }^{53}$. Recentemente, um estudo comparou o efeito da contracepção combinada oral com a contracepção combinada vaginal sobre as variáveis hemostáticas e observou que em um período curto de tempo, a hemostasia era alterada pela presença do EE, independentemente da via de administração ${ }^{54}$. Entretanto, ainda são necessários estudos para avaliar o risco de TEV com este contraceptivo vaginal.

Os injetáveis combinados provocaram menor impacto na hemostasia que as preparações orais ${ }^{55}$, diferentemente de outras formulações não-orais (adesivo e anel), provavelmente porque possuem estrogênios naturais (valerato de estradiol ou cipionato de estradiol) em sua composição, ao invés do EE. Em termos de risco para trombose, um trabalho com amostra pequena para este desfecho mostrou risco pequeno ou ausente de TEV, infarto agudo do miocárdio e acidente vascular cerebral $^{56}$, porém ainda não temos uma resposta definitiva se os combinados injetáveis apresentam, de fato, ausência de risco para TEV.

\section{Rastreamento universal de trombofilias prévio à hormonioterapia}

Acredita-se que os episódios de TEV sejam multicausais pela combinação de predisposição genética com fatores ambientais (puerpério, viagem prolongada, uso de COC, obesidade etc.). As trombofilias (tendência genética ou adquirida para trombose venosa) aumentam o risco basal da paciente para TEV, potencializando o efeito trombogênico dos COCs ou da TH ${ }^{9}$. O grau de potencialização vai depender do tipo de trombofilia: há trombofilias que aumentam em três vezes o risco de TEV (heterozigose para mutação do gene da protrombina G20210A), enquanto outras chegam a aumentar em 50 a 80 vezes este risco (homozigose para fator $\mathrm{V}$ de Leiden) ${ }^{9}$.

Porém, cerca da metade dos episódios de TEV são idiopáticos, ou seja, aqueles sem etiologia (hereditária ou adquirida) conhecida para o evento. A freqüência de trombofilias hereditárias conhecidas na população geral é baixa (0,02 a 0,5/1.000 pessoas), com exceção do fator $\mathrm{V}$ de Leiden heterozigoto, hiperhomocisteinemia e 
mutação do gene da protrombina G20210A, que apresentam prevalência máxima de $6 \%$ na população ${ }^{57}$.

Assim, o rastreamento universal para trombofilias antes da prescrição de um COC não é recomendado, uma vez que não é clinicamente viável e nem custo efetivo ${ }^{7,58}$. Soma-se a isto o fato de que o achado de uma trombofilia em assintomáticas não indica que a paciente terá TEV, apenas que tem maior risco para a ocorrência destes episódios. Da mesma forma, a ausência de diagnóstico de trombofilia conhecida também não exclui que não existam causas genéticas ainda não conhecidas que coloquem a paciente em um patamar de maior risco para TEV.

Assim, é necessária uma anamnese cuidadosa antes da prescrição do COC, buscando identificar fatores de risco adicionais para TEV. Os principais fatores a serem pesquisados, em mulheres no menacme, são história pessoal ou familiar (parentes de primeiro grau) de TEV, obesidade (IMC $\geq 30 \mathrm{~kg} / \mathrm{m}^{2}$ ) e presença de doenças crônicas associadas a TEV ${ }^{9,14}$. A obesidade aumenta em 2,7 a 4,6 vezes o risco para desenvolver trombose ${ }^{1,9}$, enquanto a história familiar de TEV garante um acréscimo de 2,5 vezes neste risco ${ }^{59}$. Uma história pessoal prévia de TEV já contra-indica o uso de estrogênio, seja para contracepção ou para TH no climatério.

\section{Considerações finais}

Normalmente, os eventos tromboembólicos ocorrem dentro do primeiro ano de uso do contraceptivo hormonal e da TH, especialmente após o quarto mês do início do uso $^{1,6,7}$. Porém, após um ano, o tempo de uso de COC não altera o risco para TEV, não havendo, dessa forma, risco acumulado com o passar dos anos ${ }^{1,6}$. Assim, trocas desnecessárias de contraceptivo hormonal combinado podem elevar o risco para TEV em cada troca ${ }^{18}$.

Não há evidências seguras de que o uso contínuo de COC apresente o mesmo risco para TEV que o uso de $\mathrm{COC}$ em regime habitual com pausa de sete dias; desta maneira, em pacientes de maior risco para TEV, esta conduta deve ser evitada.

Recentemente, a síndrome metabólica (obesidade centrípeta; elevação da pressão arterial, da glicemia de jejum e dos triglicérides; redução do HDL colesterol), que é associada a doença aterosclerótica cardiovascular com risco de trombose arterial ${ }^{60}$, parece também estar associada a aumento de risco para TEV. Este risco é duas vezes maior do aquele observado entre não portadores de síndrome metabólica ${ }^{61}$. Esta informação é importante na individualização do contraceptivo das portadoras de síndrome dos ovários policísticos que também apresentem síndrome metabólica concomitante.
Por último, o ginecologista deve ficar atento às informações que recebe sobre risco de trombose de um contraceptivo hormonal, pois, para uma conclusão segura, precisamos de uma amostra grande (já que a prevalência de trombose no menacme é muito baixa, de acordo com a Tabela 3) e de um longo tempo de observação.

\section{Resumo das recomendações}

- Para contracepção, o risco de trombose é maior em preparações com dose de EE superior ou igual a 50 mcg. Não se sabe se doses ultra-baixas (20$15 \mathrm{mcg}$ ) reduzem o risco de TEV comparadas à dose de $30 \mathrm{mcg}$;

- Para TH, sempre usar a menor dose de estrogênio capaz de promover o alívio dos sintomas climatéricos. O EEC tem maior risco de TEV que os estrogênios esterificados (não disponíveis no Brasil), porém não se sabe se o risco de TEV associado ao uso do 17-beta-estradiol ou do valerato de estradiol é inferior ao do EEC;

- Os progestagênios alteram o risco de TEV de um COC, sendo que as evidências atuais sugerem que aqueles que contêm o levonorgestrel possuem o menor risco de TEV, pois as evidências demonstram que quanto mais androgênico um progestagênio associado ao EE, menor o risco de TEV em contracepção. Para TH, não há conclusões definitivas de qual progestagênio é o mais seguro em termos de risco para TEV; há evidências iniciais de que a progesterona micronizada e os derivados pregnanos possam apresentar menor risco para TEV;

- Os contraceptivos apenas de progestagênio não estão associados a risco de TEV, sendo desta forma indicados para pacientes de risco para TEV ou história pessoal prévia de TEV;

- Apesar de ser um progestagênio, não há comprovação de que a tibolona não altere o risco de TEV, não sendo indicada para pacientes de risco para TEV ou história pessoal prévia de TEV;

- A via de administração transdérmica de hormônio em TH no climatério parece estar associada a um menor risco de TEV quando comparada à via oral;

- Já a via não-oral (transdérmica ou vaginal) de administração hormonal de contracepção combinada não difere da via oral no risco de TEV, não sendo recomendada para pacientes de risco para TEV ou história pessoal prévia de TEV;

- O risco de TEV aumenta após quatro meses de uso de um contraceptivo e não é cumu- 
lativo com o tempo de uso; assim, evitar trocas desnecessárias de contraceptivo, sem indicação clínica;

- Não há nenhuma evidência consistente favorável ao rastreamento universal de trombofilias previamente à prescrição de contraceptivo hormonal combinado. Recomenda-se, previamente à prescrição de um contraceptivo hormonal combinado, procurar fatores de risco para TEV, como obesidade e história familiar para TEV;
- Por último, devemos lembrar que a gravidez está associada a um risco mais elevado de TEV do que qualquer formulação contraceptiva combinada, mas, ao considerarmos que há milhões de usuárias de contracepção hormonal, devemos escolher, sempre que possível, a opção com menor freqüência de eventos adversos sérios, como o TEV. Como responsável pela prescrição da hormonioterapia, o ginecologista deve conhecer estes riscos para poder contribuir da melhor forma para a saúde de sua paciente.

\section{Referências}

1. Venous thromboembolic disease and combined oral contraceptives: results of international multicentre case-control study. World Health Organization Collaborative Study of Cardiovascular Disease and Steroid Hormone Contraception. Lancet. 1995;346(8990):1575-82.

2. World Health Organization. Effect of different progestagens in low oestrogen oral contraceptives on venous thromboembolic disease. World Health Organization Collaborative Study of Cardiovascular Disease and Steroid Hormone Contraception. Lancet. 1995;346(8990):1582-8.

3. Khader YS, Rice J, John L, Abueita O. Oral contraceptives use and the risk of myocardial infarction: a meta-analysis. Contraception. 2003;68(1):11-7.

4. Girolami A, Scandellari R, Tezza F, Paternoster D, Girolami B. Arterial thrombosis in young women after ovarian stimulation: case report and review of the literature. J Thromb Thrombolysis. 2007;24(2): 169-74.

5. Dahlbäck B. Blood coagulation. Lancet. 2000;355(9215):1627-32.

6. Rosendaal FR, Van Hylckama Vlieg A, Tanis BC, Helmerhorst FM. Estrogens, progestogens and thrombosis. J Thromb Haemost. 2003; 1 (7): 1371-80.

7. Rosendaal FR, Helmerhorst FM, Vandenbroucke JP. Female hormones and thrombosis. Arterioscler Thromb Vasc Biol. 2002;22(2):201-10.

8. Mammen EF. Oral contraceptive pills and hormonal replacement therapy and thromboembolic disease. Hematology Oncol Clin North Am. 2000; 14(5):1045-59.

9. Rosendaal FR. Venous thrombosis: the role of genes, environment, and behavior. Hematology Am Soc Hematol Educ Program. $2005 ; 1-12$

10. Hulley S, Grady D, Bush T, Furberg C, Herrington D, Riggs B, et al. Randomized trial of estrogen plus progesterone for secondary prevention of coronary heart disease in postmenopausal women. Heart and Estrogen/progestin Replacement Study (HERS) Research Group. JAMA. 1998;280(7):605-13

11. Grady D, Wenger NK, Herrington D, Khan S, Furberg C, Hunninghake $D$, et al. Postmenopausal hormone therapy increases risk for venous thromboembolic disease. The Heart and Estrogen/progestin Replacement Study. Ann Intern Med. 2000;132(9):689-96.

12. Rossouw JE, Anderson GL, Prentice RL, LaCroix AZ, Kooperberg C, Stefanick ML. Risks and benefits of estrogen plus progestin in healthy postmenopausal women: principal results From the
Women's Health Initiative randomized controlled trial. JAMA. 2002;288(3):321-33

13. Anderson GL, Limacher M, Assaf AR, Bassford T, Beresford SA, Black $\mathrm{H}$, et al. Effects of conjugated equine estrogen in postmenopausal women with hysterectomy: the Women's Health Initiative randomized controlled trial. JAMA. 2004;291(14):1701-12.

14. Lowe GD. Venous and arterial thrombosis: epidemiology and risk factors at various ages. Maturitas. 2004;47(4):259-63.

15. Smith NL, Heckbert SR, Lemaitre RN, Reiner AP, Lumley $T$, Weiss NS, et al. Esterified estrogens and conjugated equine estrogens and the risk of venous thrombosis. JAMA. 2004;292(13):1581-7.

16. Smith NL, Heckbert SR, Doggen CJ, Lemaitre RN, Reiner AP, Lumley $\mathrm{T}$, et al. The differential association of conjugated equine estrogen and esterified estrogen with activated protein $C$ resistance in postmenopausal women. J Thromb Haemost. 2006;4(8):1701-6.

17. Smith NL, Heckbert SR, Lemaitre RN, Reiner AP, Lumley $T$, Rosendaal FR, et al. Conjugated equine estrogen, esterified estrogen, prothrombotic variants, and the risk of venous thrombosis in postmenopausal women. Arterioscler Thromb Vasc Biol. 2006;26(12):2807-12.

18. Kemmeren JM, Algra A, Grobbee DE. Third generation oral contraceptives and risk of venous thrombosis: meta-analysis. BM. 2001;323(7305): 131-4.

19. Schindler $A E$, Campagnoli C, Druckmann R, Huber J, Pasqualini JR, Schweppe KW, et al. Classification and pharmacology of progestins. Maturitas. 2003;46 Suppl 1:S7-S16.

20. Herkert O, Kuhl H, Sandow J, Busse R, Schini-Kerth VB. Sex steroids used in hormonal treatment increase vascular procoagulant activity by inducing thrombin receptor (PAR-1) expression: role of the glucocorticoid receptor. Circulation. $2001 ; 104(23): 2826-31$.

21. Rosing J, Tans G, Nicolaes GA, Thomassen MC, van Oerle R, van der Ploeg PM, et al. Oral contraceptives and venous thrombosis: different sensitivities to activated protein $C$ in women using second- and third-generation oral contraceptives. $\mathrm{Br} J$ Haematol. $1997 ; 97(1): 233-8$

22. Kemmeren JM, Algra A, Meijers JC, Tans G, Bouma BN, Curvers J, et al. Effect of second- and third-generation oral contraceptives on the protein $C$ system in the absence or presence of the factor VLeiden mutation: a randomized trial. Blood. 2004; 103(3):927-33. 
23. Conard J. Biological coagulation findings in third-generation oral contraceptives. Hum Reprod Uptade. 1999;5(6):672-80.

24. Tans G, Curvers J, Middeldorp S, Thomassen MC, Meijers $J C$, Prins $M H$, et al. A randomized cross-over study on the effects of levonorgestrel- and desogestrel-containing oral contraceptives on the anticoagulant pathways. Thromb Haemost. 2000;84(1):15-21.

25. Tans $G$, van Hylckama Vlieg A, Thomassen MC, Curvers J, Bertina RM, Rosing J, et al. A randomized cross-over study on the effects of levonorgestrel- and desogestrel-containing oral contraceptives on the anticoagulant pathways. Br J Haematol. 2003; 122(3):465-70.

26. Vasilakis-Scaramozza C, Jick H. Risk of venous thromboembolism with cyproterone or levonorgestrel contraceptives. Lancet. 2001;358(9291):1427-9.

27. Seaman HE, de Vries CS, Farmer RD. The risk of venous thromboembolism in women prescribed cyproterone acetate in combination with ethinyl estradiol: a nested cohort analysis and case-control study. Hum Reprod. 2003;18(3):522-6.

28. Alhenc-Gelas M, Plu-Bureau G, Guillonneau S, Kirzin JM, Aiach $M$, Ochat $N$, et al. Impact of progestagens on activated protein $C(A P C)$ resistance among users of oral contraceptives. J Thromb Haemost. 2004;2(9): 1594-600.

29. van Vliet HA, Winkel TA, Noort I, Rosing J, Rosendaal FR. Prothrombotic changes in users of combined oral contraceptives containing drospirenone and cyproterone acetate. J Thromb Haemost. 2004;2(1 1):2060-2.

30. van Vliet HA, Frolich M, Christella M, Thomassen LG, Doggen CJ, Rosendaal FR, et al. Association between sex hormone-binding globulin levels and activated protein $C$ resistance in explaining the risk of thrombosis in users of oral contraceptives containing different progestogens. Hum Reprod. 2005;20(2):563-8.

31. Odlind V, Milsom I, Persson I, Victor A. Can changes in sex hormone binding globulin predict the risk of venous thromboembolism with combined oral contraceptive pills? Acta Obstet Gynecol Scand. 2002;81 (6):482-90.

32. Klipping C, Marr J. Effects of two combined oral contraceptives containing ethinyl estradiol 20 microg combined with either drospirenone or desogestrel on lipids, hemostatic parameters and carbohydrate metabolism. Contraception. 2005;71(6):409-16.

33. Dinger JC, Heinemann LA, Kühl-Habich D. The safety of a drospirenone-containing oral contraceptive: final results from the European Active Surveillance Study on oral contraceptives based on 142,475 women-years of observation. Contraception. 2007;75(5):344-54.

34. World Health Organization. Cardiovascular disease and steroid hormone contraception. Report of a WHO Scientific group. Geneva: World Health Organization; 1998. (WHO Technical Report Series, 877).

35. Canonico M, Oger E, Plu-Bureau G, Conard J, Meyer G, Lévesque $H$, et al. Hormone therapy and venous thromboembolism among postmenopausal women: impact of the route of estrogen administration and progestogens: the ESTHER study. Circulation. 2007; 1 15(7):840-5.

36. Conard J, Plu-Bureau G, Bahi N, Horellou MH, Pelissier C, Thalabard JC. Progestogen-only contraception in women at high risk of venous thromboembolism. Contraception. 2004;70(6):437-41.

37. World Health Organization. Medical eligibility criteria for contraceptive use. $3^{\text {rd }}$ ed. Geneva: World Health Organization; 2004.

38. Winkler UH, Howie H, Buhler K, Korver T, Geurts TB, Coelingh Bennink HJ. A randomized controlled double-blind study of the effects on hemostasis of two progestogen-only pills containing
75 microgram desogestrel or 30 microgram levonorgestrel. Contraception. 1998;57(6):385-92.

39. Dorflinger L. Metabolic effects of implantable steroid contraceptives for women. Contraception. 2002;65(1):47-62.

40. International Collaborative Post-Marketing Surveillance of Norplant. Post-marketing surveillance of Norplant contraceptive implants: II. Non-reproductive health. Contraception. 2001;63(4):187-209.

41. Meirik O, Farley TM, Sivin I. Safety and efficacy of levonorgestrel implant, intrauterine device, and sterilization. Obstet Gynecol. $2001 ; 97(4): 539-47$

42. Egberg N, van Beek A, Gunnervik C, Hulkko S, Hirvonen E, LarssonCohn $U$, et al. Effects on hemostatic system and liver function in relation to Implanon ${ }^{\circledast}$ and Norplant ${ }^{\circledR}$. A prospective randomized clinical trial. Contraception. 1998;58(2):93-8.

43. Lindqvist PG, Rosing J, Malmquist A, Hillarp A. Etonogestrel implant use is not related to hypercoagulable changes in anticoagulant system. J Thromb Haemost. 2003;1 (3):601-2.

44. Vieira CS, Ferriani RA, Garcia AA, Gomes MK, Azevedo GD, Silva de Sá MF. Transitory reduction of platelet aggregation with the use of etonogestrel implant in healthy women. Thromb Haemost. 2005;94(3):682-3

45. Vieira CS, Ferriani RA, Garcia AA, Pintão MC, Azevedo GD, Gomes MK, et al. Use of the etonogestrel-releasing implant is associated with hypoactivation of the coagulation cascade. Hum Reprod. 2007;22(8):2196-201.

46. Eilertsen AL, Qvigstad E, Andersen TO, Sandvik L, Sandset PM. Conventional-dose hormone therapy (HT) and tibolone, but not lowdose HT and raloxifene, increase markers of activated coagulation. Maturitas. 2006;55(3):278-87

47. Eilertsen AL, Sandvik L, Mowinckel MC, Andersen TO, Qvigstad $E$, Sandset PM. Differential effects of conventional and low dose oral hormone therapy $(\mathrm{HT})$, tibolone, and raloxifene on coagulation and fibrinolysis. Thromb Res. 2007;120(3):371-9.

48. Eilertsen AL, Liestol S, Mowinckel MC, Hemker HC, Sandset PM. Differential impact of conventional and low-dose oral hormone therapy $(\mathrm{HT})$, tibolone and raloxifene on functionality of the activated protein C system. Thromb Haemost. 2007;97(6):938-43.

49. Skouby SO, Sidelmann JJ, Nilas L, Jespersen J. A comparative study of the effect of continuous combined conjugated equine estrogen plus medroxyprogesterone acetate and tibolone on blood coagulability. Hum Reprod. 2007;22(4): 1 186-91

50. Scarabin PY, Oger E, Plu-Bureau G; Estrogen and ThromboEmbolism Risk Study Group. Differential association of oral and transdermal oestrogen replacement therapy with venous thromboembolism risk. Lancet. 2003;362(9382):428-32.

51. Food and Drug Administration. Center for Drug Evaluation and Research. Ortho Evra (norelgestromin/ethinyl estradiol) Information [text on the Internet]. 2006 [cited 2007 Jul 20]. Available from: http:// www.fda.gov/cder/drug/infopage/orthoevra/default.htm

52. Jick SS, Kaye JA, Russmann S, Jick H. Risk of nonfatal venous thromboembolism in women using a contraceptive transdermal patch and oral contraceptives containing norgestimate and $35 \mathrm{Ag}$ of ethinyl estradiol. Contraception. 2006;73(3):223-8.

53. Magnusdóttir EM, Bjarnadóttir RI, Onundarson PT, Gudmundsdóttir BR, Geirsson RT, Magnusdóttir SD, et al. The contraceptive vaginal ring (NuvaRing) and hemostasis: a comparative study. Contraception. 2004;69(6):461-7.

54. Sitruk-Ware R, Plu-Bureau G, Menard J, Conard J, Kumar S, Thalabard JC, et al. Effects of oral and transvaginal ethinyl estradiol on hemostatic factors and hepatic proteins in a randomized, crossover study. J Clin Endocrinol Metab. 2007;92(6):2074-9. 
55. United Nations Development Programme/United Nations Population Fund/World Health Organization/World Bank Special Programme of Research, Development and Research Training in Human Reproduction, Task Force on Long-acting Systemic Agents for Fertility Regulation. Comparative study of the effects of two once-a-month injectable contraceptives (Cyclofem ${ }^{\circledR}$ and Mesigyna ${ }^{\circledR}$ ) and one oral contraceptive (OrthoNovum $1 / 35^{\circledR}$ ) on coagulation and fibrinolysis. Contraception. 2003;68(3):159-76.

56. World Health Organization Collaborative Study of Cardiovascular Disease and Steroid Hormone Contraception. Cardiovascular disease and use of oral and injectable progestogenonly contraceptives. Contraception. 1998;57(5):315-24.

57. Pabinger I, Vormittag R. Thrombophilia and pregnancy outcomes. J Thromb Haemost. 2005;3(8):1603-10.

58. Wu O, Robertson L, Twaddle S, Lowe GD, Clark P, Greaves M, et al. Screening for thrombophilia in high-risk situations: systematic review and cost-effectiveness analysis. The Thrombosis: Risk and Economic Assessment of Thrombophilia Screening (TREATS) study. Health Technol Assess. 2006;10(11):1-110.

59. Bloemenkamp KW, Rosendaal FR, Büller HR, Helmerhorst FM, Colly LP, Vandenbroucke JP. Risk of venous thrombosis with use of current low-dose oral is not explained by diagnostic suspicion and referral bias. Arch Intern Med. 1999;159(1):65-70.

60. Grundy SM, Cleeman JI, Daniels SR, Donato KA, Eckel RH, Franklin BA, et al. Diagnosis and management of the metabolic syndrome: an American Heart Association/National Heart, Lung, and Blood Institute Scientific Statement. Circulation. 2005; $112(17): 2735-52$.

61. Ay C, Tengler T, Vormittag R, Simanek R, Dorda W, Vukovich T, et al. Venous thromboembolism-a manifestation of the metabolic syndrome. Haematologica. 2007;92(3):374-80. 